\title{
Instability of the one-texture universe
}

\author{
Xuelei Chen* \\ Department of Physics, Columbia University, 538 West 120th Street, New York, New York 10027 \\ Mark Hindmarsh ${ }^{\dagger}$ \\ Centre for Theoretical Physics, University of Sussex, Falmer, Brighton BN1 9QH, United Kingdom \\ Marc Kamionkowski ${ }^{\ddagger}$ \\ Department of Physics, Columbia University, 538 West 120th Street, New York, New York 10027 \\ Andrew R. Liddle ${ }^{\S}$ \\ Astronomy Centre, University of Sussex, Falmer, Brighton BN1 9QH, United Kingdom
}

(Received 31 January 1997)

\begin{abstract}
The one-texture universe, introduced by Davis in 1987, is a homogeneous mapping of a scalar field with an $S^{3}$ vacuum into a closed universe. It has long been known to mathematicians that such solutions, although static, are unstable. We show by explicit construction that there are four degenerate lowest modes which are unstable, corresponding to collapse of the texture towards a single point, in the case where gravitational back reaction is neglected. We discuss the instability time scale in both static and expanding space-times; in the latter case it is of order of the present age of the universe, suggesting that, though unstable, the one-texture universe could survive to the present. The cosmic microwave background constrains the initial magnitude of this unstable perturbation to be less than $\sim 10^{-3}$. [S0556-2821(97)07416-X]
\end{abstract}

PACS number(s): 98.80.Hw, 11.27.+d, 95.35.+d

\section{INTRODUCTION}

A texture is a topological defect which arises in scalar field theories with a spontaneously broken global symmetry, when the vacuum manifold $M$ has a nontrivial third homotopy group $\pi_{3}$. The simplest such manifold is the threesphere $S^{3}$. In a spatially flat universe, the texture is known to be unstable to collapse, and indeed this property is crucial for the texture model of structure formation in the universe [1]. The one-texture universe (which predates and has nothing to do with the texture scenario for structure formation), introduced by Davis [2], considers instead a closed universe, itself with $S^{3}$ topology, and arises when one maps the vacuum manifold directly onto the configuration space. This gives a nontrivial homogeneous solution to the equations of motion, the scalar field possessing an energy density associated with its spatial gradients. This scalar-field configuration has an equation of state $p=-\rho / 3$ and gives rise to a term in the Friedmann equation which scales as $a^{-2}$ with the scale factor $a$ of the universe. Recently, Kamionkowski and Toumbas [3] showed that a universe with a matter density less than unity can be closed (and consistent with current observations) with the inclusion of matter with $p=-\rho / 3$.

Aware that textures collapse in flat space through shrinking, Davis considered a particular shrinking ansatz for the one-texture universe, and showed that it was stable to this particular kind of perturbation [2]. He concluded from this

\footnotetext{
*Electronic address: xuelei@phys.columbia.edu

${ }^{\dagger}$ Electronic address: m.b.hindmarsh@sussex.ac.uk

†Electronic address: kamion@phys.columbia.edu

${ }^{\S}$ Electronic address: a.liddle@ @ussex.ac.uk
}

that the one-texture universe was stable. However, this does not correspond to a complete stability analysis. Shortly after we began looking at this problem, it was pointed out to us by Durrer that in fact the answer was already in the mathematics literature [4], predating even the original Davis paper. The one-texture universe is unstable. Indeed, there is no nonconstant stable map from any compact manifold into $S^{3}$ [4].

However, the results in the mathematics literature apply, strictly speaking, only to a static universe. It is well known that the expansion of the universe slows the growth of density perturbations and similarly that the expansion of a deflagration bubble slows the growth of hydrodynamic instabilities in the bubble wall [5]. It is therefore of interest to see whether the growth of instabilities in the one-texture universe are slowed by the expansion of the universe, and to evaluate the time scale for instability.

In this paper, we consider the instability by explicit construction. We show that there are four degenerate lowest modes which are unstable, and analyze their growth rate in both static and expanding space-times.

In the next section, we present some preliminaries regarding the closed universe and the scalar field theory. In Sec. III, we first review the texture scalar field configuration. We then find an unstable mode and analyze its growth rate in a static universe, in a radiation-, matter-, texture-, and cosmologicalconstant dominated universe, and in a universe with comparable matter and texture energy densities. The growth rate in an expanding universe is slowed compared with the exponential rate in a static universe, and during inflation, the instability is frozen. The instability time scale is comparable to the age of the universe. In Sec. IV, we perform a general perturbation analysis and find that there are four degenerate modes including the one found in Sec. III. There are also six 
zero modes corresponding to rotations and translations. All other higher modes are stable and decay in an expanding universe. We conclude in Sec. V and argue that isotropy of the cosmic microwave background only constrains the magnitude of the initial unstable mode to be less than $\sim 10^{-3}$.

\section{PRELIMINARIES}

The space-time metric for a closed universe can be written as

$$
d s^{2}=a^{2}(\tau)\left[-d \tau^{2}+d \xi^{2}+\sin ^{2} \xi\left(d \theta^{2}+\sin ^{2} \theta d \phi^{2}\right)\right],
$$

with

$$
0 \leqslant \xi<\pi, \quad 0 \leqslant \theta<\pi, \quad 0 \leqslant \phi<2 \pi,
$$

where $\tau$ is the conformal time and $a(\tau)$ the scale factor.

We consider a theory of four real scalar fields with an $\mathrm{O}(4)$ global symmetry, spontaneously broken to $\mathrm{O}(3)$ by a suitable potential. The vacuum manifold for the scalar field is therefore $S^{3}$. If we consider the long-wavelength modes, the massive degree of freedom (i.e., the $\sigma$-meson in a linear $\sigma$ model) would not be excited, and the dynamics are approximately those of a nonlinear sigma model with this target space. The action is therefore

$$
S=\frac{v^{2}}{2} \int d^{4} x \sqrt{|g|} G_{A B}(X) \partial_{\mu} X^{A}(x) \partial_{\nu} X^{B}(x) g^{\mu \nu}(x),
$$

where $X^{A}(A=1,2,3)$ are coordinates on the target space $M$, which has metric $G_{A B}$, and $v$ is the vacuum expectation of the scalar field. We will also use polar coordinates for $M$, namely $\Xi, \Theta, \Phi$, so that $G_{A B}=\operatorname{diag}\left(1, \sin ^{2} \Xi\right.$, $\left.\sin ^{2} \Xi \sin ^{2} \Theta\right)$. Their ranges are as with $\xi, \theta$, and $\phi$, respectively.

The equations of motion for $X^{A}$ are

$$
\frac{1}{\sqrt{|g|}} \partial_{\mu}\left(\sqrt{|g|} g^{\mu \nu} \partial_{\nu} X^{A}\right)+\Gamma_{B C}^{A}(X) \partial_{\mu} X^{B} \partial_{\nu} X^{C} g^{\mu \nu}(x)=0,
$$

where $\Gamma_{B C}^{A}$ are the Christoffel symbols of the metric $G_{A B}$ on $M$. Solutions to these equations were called harmonic maps by Misner [6].

In our stability analysis we will need to consider the eigenmodes of small perturbations around static solutions $X^{A}=f^{A}$ to these equations, for which we will write $X^{A}=f^{A}+\epsilon n^{A}$. It is possible to define a covariant derivative for $n^{A}$, by

$$
n_{; \mu}^{A}=\partial_{\mu} n^{A}+\left(\Gamma_{B C}^{A} \partial_{\mu} f^{C}\right) n^{B} .
$$

The equation for linear perturbations around $f^{A}$ may then be written

$$
n_{; \mu}^{A} ; \mu+\left(R_{C B D}^{A} \partial_{\mu} f^{C} \partial^{\mu} f^{D}\right) n^{B}=0,
$$

where $R_{C B D}^{A}$ is the Riemann curvature of the sigma model target space $M$.

Before solving the perturbation equation in its generality, we describe the one-texture universe and examine a simple perturbation around it. The lowest eigenvalue solution of this perturbation equation will turn out to be one of a set of four degenerate unstable modes.

\section{THE ONE-TEXTURE UNIVERSE AND A PERTURBATION}

The trivial solution has the symmetry breaking pointing in the same direction (say $\Xi=\Theta=\Phi=0$ ) at all points of space. By contrast, the one-texture universe corresponds to the solution [2]

$$
\Xi=\xi, \quad \Theta=\theta, \quad \Phi=\phi .
$$

That this is a solution is clear once we realize that $e_{i}^{A} \equiv \partial_{i} f^{A}=\delta_{i}^{A}$, where $i$ and $j$ are spatial indices. Then it follows that $G^{A B}=\delta_{i}^{A} \delta_{j}^{B} g^{i j}$, and Eq. (3) becomes the wellknown geometric identity

$$
\frac{1}{\sqrt{|G|}} \partial_{B}\left(\sqrt{|G|} G^{B A}\right)+\Gamma_{B C}^{A} G^{B C}=0 .
$$

We now consider a linear perturbation to the radial component which is a function of $\xi$ only. This is the simplest guess for an unstable mode, and as we shall see turns out to be correct. We therefore let $\Xi=\xi+\delta(\xi, \tau)$. An equation of motion for $\delta(\xi, \tau)$ can be found by plugging directly into Eq. (5). It is easier to vary the action, which for a perturbation of this form is

$$
\begin{gathered}
S \propto \int d \tau d \xi a^{2} \sin ^{2} \xi\left[-(\partial \Xi / \partial \tau)^{2}+(\partial \Xi / \partial \xi)^{2}\right. \\
\left.+2 \sin ^{2} \Xi \csc ^{2} \xi\right] .
\end{gathered}
$$

Doing so, we find

$$
\begin{gathered}
\left(\frac{d^{2}}{d \xi^{2}}+2 \frac{\cos \xi}{\sin \xi} \frac{d}{d \xi}+4-\frac{2}{\sin ^{2} \xi}\right) \delta(\xi, \tau) \\
=\left(\frac{d^{2}}{d \tau^{2}}+2 \frac{\dot{a}}{a} \frac{d}{d \tau}\right) \delta(\xi, \tau),
\end{gathered}
$$

where overdot is a derivative with respect to the conformal time $\tau$. As usual, we look for separable solutions $\delta(\xi, \tau)=\delta(\xi) f(\tau)$. Let us concentrate first on the spatial eigenmodes, which satisfy

$$
\left(\frac{d^{2}}{d \xi^{2}}+2 \frac{\cos \xi}{\sin \xi} \frac{d}{d \xi}+4-\frac{2}{\sin ^{2} \xi}\right) \delta(\xi)=-\omega^{2} \delta(\xi) .
$$

This can in fact be brought into Schrödinger equation form by a change of independent variable to $u(\xi)=\delta(\xi) \sin \xi$, yielding

$$
-\frac{d^{2} u}{d \xi^{2}}+\frac{2}{\sin ^{2} \xi} u=\left(\omega^{2}+5\right) u
$$

The first two unnormalized eigenmodes are $u_{1}(\xi)=\sin ^{2} \xi$ and $u_{2}(\xi)=\sin ^{2} \xi \cos \xi$, with eigenvalues -1 and 4 , respectively. These form the first two elements of a series 


$$
u_{n}(\xi)=\sin ^{2} \xi C_{n-1}^{(2)}(\cos \xi) \quad(n \geqslant 1)
$$

where $C_{m}^{(\lambda)}(t)$ are Gegenbauer polynomials of degree $m$ [7]. The eigenvalue corresponding to the $n$th eigenfunction is $\omega^{2}=n(n+2)-4$.

The stability (or otherwise) now follows from the time eigenfunctions, which depend on the behavior of the scale factor. They are solutions to the equation

$$
\left(\frac{d^{2}}{d \tau^{2}}+2 \frac{\dot{a}}{a} \frac{d}{d \tau}\right) f(\tau)=-\omega^{2} f(\tau)
$$

which we mention in passing is precisely the equation for the amplitude of gravitational-wave modes [8], though there the range of permitted eigenvalues is different.

\section{A. The static universe}

In this case the time eigenfunctions are simply

$$
f(\tau) \propto \exp ( \pm i \omega \tau)
$$

The higher spatial eigenmodes, with positive $\omega^{2}$, are oscillatory. However, the lowest eigenmode has negative $\omega^{2}$, and hence corresponds to an exponentially growing instability. This provides an explicit confirmation of the mathematical result of Ref. [4].

The instability corresponds to the spatial gradients concentrating towards one of the poles (which one depending on whether the sign of the perturbation is positive or negative). Presumably by analogy to the spatially flat case, once the winding is sufficiently concentrated the texture will be pulled away from its vacuum manifold and the topological charge disappears.

\section{B. Radiation domination}

A radiation dominated universe has $a(\tau) \propto \tau$. The time eigenmodes are then

$$
f(\tau) \propto \frac{\exp ( \pm i \omega \tau)}{\tau} .
$$

We see that the condition for instability remains $\omega^{2}<0$.

\section{Matter domination}

Here $a(\tau) \propto \tau^{2}$. The time eigenmodes for positive $\omega^{2}$ are now given in terms of spherical Bessel functions [9]

$$
f(\tau)=\alpha_{1} \frac{j_{1}(\omega \tau)}{\tau}+\alpha_{2} \frac{y_{1}(\omega \tau)}{\tau}
$$

where $\alpha_{1}$ and $\alpha_{2}$ are constants. For negative $\omega^{2}$ these become modified spherical Bessel functions; the linearly independent mode of the first kind diverges at late times and gives the instability.

\section{Texture-dominated universe without back reaction}

In a texture-dominated closed universe without other matter, $a(\tau) \propto \exp \left(\tau / \sqrt{\Omega_{T}-1}\right)$, where the texture energy density

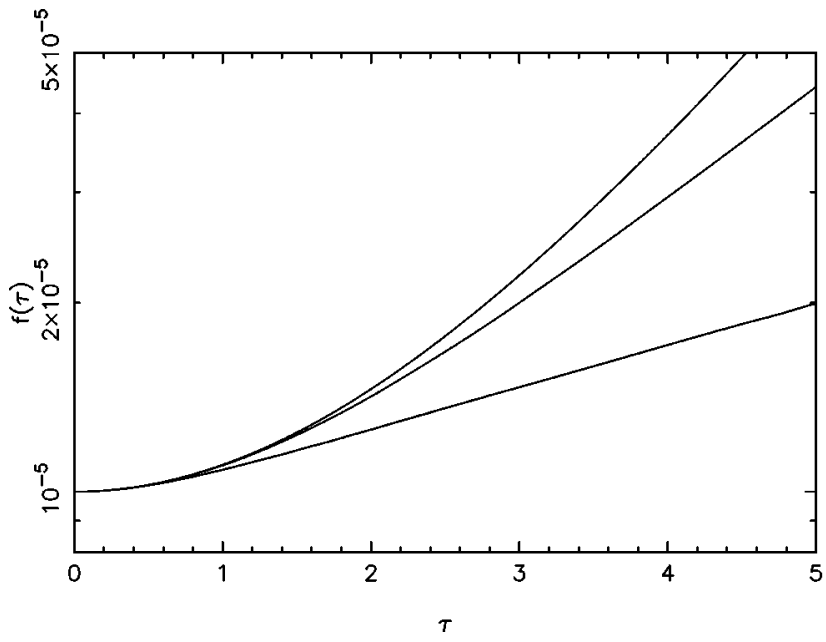

FIG. 1. The growth of the unstable perturbation in a one-texture universe with matter, shown, from top to bottom, for $\alpha_{0}=0.1,1$, and 10 .

as a fraction of the critical density, $\Omega_{T}$, is a constant. The solution to Eq. (14) is of the form

$$
f(\tau) \propto \exp (\beta \tau) ; \quad \beta=-\frac{1 \pm \sqrt{1-\omega^{2}\left(\Omega_{T}-1\right)}}{\sqrt{\Omega_{T}-1}} .
$$

For the $\omega^{2}=-1$ mode, the solutions are just a growing and a decaying exponential. For positive $\omega^{2}$, the constant $\beta$ is complex, with sine and cosine oscillations superimposed on an exponential decay.

The more interesting solution is one where there is still an appreciable (nonrelativistic) matter density, such as in Ref. [3]. Then, denoting present values with the subscript ' 0 ,', the expansion rate is

$$
\frac{a(\tau)}{a_{0}}=\frac{\Omega_{M, 0}}{2\left(1-\Omega_{M, 0}\right)}\left[\cosh \left(\alpha_{0}^{1 / 2} \tau\right)-1\right],
$$

where the constant $\alpha_{0}$ is given by

$$
\alpha_{0}=\frac{1-\Omega_{M, 0}}{\Omega_{M, 0}+\Omega_{T, 0}-1},
$$

where $\Omega_{M}$ and $\Omega_{T}$ are the energy densities in matter and texture, respectively, in units of the critical density. For a given choice of $\Omega_{M, 0}$ and $\Omega_{T, 0}$, the present time $\tau_{0}$ is found from the requirement $a\left(\tau_{0}\right) \equiv a_{0}$, giving

$$
\tau_{0}=\alpha_{0}^{-1 / 2} \operatorname{arccosh}\left(\frac{1-\Omega_{M, 0} / 2}{\Omega_{M, 0} / 2}\right) .
$$

The corresponding equation for the time evolution of the eigenmodes does not look promising for analytical solution, but is easily solved numerically. The solution for the unstable mode is shown in Fig. 1, for three values of $\alpha_{0}$ and for a perturbation with zero initial velocity and arbitrarily chosen initial size. The late-time exponential behavior is as predicted from the case of total texture-domination $\left(\beta=\sqrt{1+\alpha_{0}}-\sqrt{\alpha_{0}}\right)$; the matter-dominated solution applies 
at early time, but matter domination does not last long enough for much growth during that phase.

Given $\Omega_{M, 0}$ and $\Omega_{T, 0}$, the value of $f\left(\tau_{0}\right)$ can be found from Fig. 1. For example, if $\Omega_{M, 0} \simeq 0.3$ (as some observations indicate), then $\Omega_{T, 0} \simeq 1.8$ if we impose the (perhaps arbitrary) condition of Ref. [3] that the cosmic microwave background (CMB) surface of last scatter be at the antipode. In this case, $\alpha_{0} \simeq 0.64, \tau_{0} \simeq 3$, and $f\left(\tau_{0}\right) / f(0) \simeq 2$. In general, the time scale of the unwinding of texture is the same as the age of the universe, which is not surprising since it is also the horizon scale. Therefore, in this low-density closed universe, any initial perturbations to the texture would have doubled by today. Note that the radiation-dominated phase preceding the matter dominance has little effect since the matter-radiation transition occurred at redshift $z \sim 10^{4}$, where $a \sim 10^{-6} a_{0}$. At that epoch the long wavelength modes had not yet entered the horizon, and so the growth of perturbations in the texture is negligible.

\section{E. An inflationary universe}

In all the above cases, the instability associated with the $\omega^{2}=-1$ mode diverges at late times. In this final case we explore a slightly different outcome. This occurs in the case of perturbations in an inflationary universe, which is dominated by vacuum energy or equivalently by a cosmological constant. We consider power-law inflation which has $a \propto t^{p}$, where $t$ is cosmic time and $p>1$ is a constant. In conformal time we have

$$
a(\tau) \propto(-\tau)^{-p /(p-1)}, \quad-\infty<\tau<0,
$$

where late times correspond to $\tau \rightarrow 0$ and where exponential inflation is recovered in the limit $p \rightarrow \infty$. The solution for positive $\omega^{2}$, in terms of fractional-order Bessel functions, is

$$
f(\tau)=\alpha_{1}(-\tau)^{\mu} J_{\mu}(-\omega \tau)+\alpha_{2}(-\tau)^{\mu} Y_{\mu}(-\omega \tau),
$$

where $\mu=p /(p-1)$ and again $\alpha_{1}$ and $\alpha_{2}$ are constants. For negative $\omega^{2}$, the Bessel functions become modified Bessel functions. In both cases, the solution of the first kind vanishes at late times, whereas the second kind "freezes out" at a constant value.

Here the distinction between stability and instability is much murkier, because of this characteristic type of behavior in inflationary universes. The positive $\omega^{2}$ modes, which would ordinarily be oscillatory about the stable solution, become frozen with a displacement away from the stable solution, while the negative $\omega^{2}$ mode, that would normally grow to divergence, also becomes frozen at late times. This interesting behavior has already been investigated for the case of gravitational-wave perturbations, which also freeze to a constant value, by Allen [10]. He describes this behavior as "global instability and local stability"; although globally the de Sitter space becomes more and more distorted as modes of higher and higher $\omega$ freeze out, the region accessible to any observer shrinks rapidly during inflation and seems to become more and more smooth. The same behavior is evident here.

Nevertheless, effectively the nature of the stability is just the same as before. Note in particular that the $\omega^{2}=-1$ mode does not die away during the late stages of inflation, but instead stays fixed; once radiation or matter domination restarts its growth will begin again, though on a long time scale since the period of inflation has stretched it to such a large physical size. Only once the Hubble length has grown again to be comparable to the size of the closed universe will the instability set in earnest.

\section{GENERAL PERTURBATION ANALYSIS}

The general solutions to Eq. (5) are in fact quite straightforward to find, as they turn out to be vector harmonics on $S^{3}$ which allows us to plunder the mathematics literature [11]. Once again, we can use the constancy of $\partial_{i} f^{A}$ to rewrite the perturbation equations as an eigenvalue problem for the vector $n^{A}$, as

$$
n_{; \mu}^{A} ; \mu=-a^{-2} \partial_{\tau}\left(a^{2} \partial_{\tau} n^{A}\right)+n_{; C ; D}^{A} G^{C D},
$$

where we use the fact that in the one-texture universe background $g_{i j}=\delta_{i}^{A} \delta_{j}^{B} G_{A B}$. Further simplification arises as the background is a three-sphere, for which

$$
R_{C B D}^{A} G^{C D}=2 \delta_{B}^{A} .
$$

Hence, when we separate the solution, the equation for the spatial eigenmodes becomes

$$
n^{A}{ }_{; C ; D} G^{C D}+2 n^{A}=-\omega^{2} n^{A} .
$$

This is an eigenvalue equation for vector harmonics on $S^{3}$, whose solutions are known [11]. They fall into three classes, $A_{A}, B_{A}$, and $C_{A}$, which are odd and even parity divergencefree, and curl-free respectively. The eigenvalues of the Laplacian for the curl-free harmonics are $[2-n(n+2)]$, with $n>0$, while for the divergence-free harmonics they are $[1-n(n+2)]$. Hence our eigenvalue problem also has three classes of solution classified by their symmetry properties under $S^{3}$ coordinate transformations, with eigenvalues

$$
\begin{gathered}
\omega_{0}^{2}=n(n+2)-4, \quad n \geqslant 1, \\
\omega_{ \pm 1}^{2}=n(n+2)-3, \quad n \geqslant 1,
\end{gathered}
$$

where the subscript 0 denotes the curl-free class, and \pm 1 the even and odd parity divergence-free class. The curl-free modes are those we found in Sec. III.

We can now see why the Gegenbauer polynomials made their appearance in Eq. (11). The curl-free vector harmonics are just the divergence of the scalar harmonics on $S^{3}$ :

$$
C_{A}(X)=\partial_{A} Y^{(n l m)}(\xi, \theta, \phi),
$$

where

$$
\begin{aligned}
Y^{(n l m)}(\xi, \theta, \phi)= & N_{n l} \sin ^{l} \xi C_{n-l}^{(l+1)}(\cos \xi) Y^{(l m)}(\theta, \phi), \\
& -l \leqslant m \leqslant l \leqslant n .
\end{aligned}
$$

[Here, $N_{n l}$ is a normalization factor and $Y^{(l m)}(\theta, \phi)$ are the usual $S^{2}$ spherical harmonics.] The relation $\partial_{t} C_{n}^{(\lambda)}(t)$ $=2 \lambda C_{n-1}^{(\lambda+1)}(t)$ then allows us to find all the $l=0$ harmonics in the direction $\partial_{\xi} X^{A}$, which amounts to solving for $\delta(\xi)$ in Eq. (11). 
Thus we see that the only unstable modes are those with $n=1$ curl-free vector harmonics. There are four of them, and the spherical one we analyzed in Sec. III corresponds $l=m=0$; there are also three anisotropic modes, which are $l=1, m=0, \pm 1$. As they have the same eigenvalue, the time eigenfunctions found in Sec. III apply to each of them. There are also six zero modes in the divergence-free class, which correspond to rotations and translations of the texture solution. The rest of the modes are stable, and correspond to propagating Goldstone bosons.

\section{CONCLUSIONS}

We have investigated in detail the nature of the instability in the one-texture universe. We have found that there are four degenerate unstable modes, and evaluated the growth rate in a radiation-, matter-, and texture-dominated universe, an inflationary universe, and a universe with similar matter and texture densities. In a static universe, the growth of the instability is exponential. As one may have expected, the growth of the instability is slowed during matter, radiation, or texture domination, and it is frozen during an inflationary epoch. Since the eigenvalue of the unstable modes is -1 , the time scale for the instability is comparable to the age of the universe. For a given texture and matter density, the growth factor can be obtained explicitly from the curves in Fig. 1. For the case of a closed universe with $\Omega_{M, 0}=0.3$ and a texture density which puts the CMB surface of last scatter at the antipode, any initial unstable perturbation would have increased by a factor of 2 .

Causality restricts the unstable modes from growing until the wavelength - in this case, the curvature radius-comes within the horizon. If the texture density is chosen so that the CMB is at the antipode, then the curvature radius today coincides with the horizon. If so, then the instability time scale is comparable to the age of the universe. If, however, the texture density is much smaller so that the curvature radius greatly exceeds the horizon today, then the instability time scale will be much longer than the age of the universe today. Strictly speaking, one should also consider the effect of gravitational back reaction. However, for most of the cases we consider here, the texture makes a negligible contribution to the total energy density, so inclusion of back reaction should not alter our results qualitatively. For the texturedominated case, back reaction may significantly affect the evolution. However, causality still restricts modes from growing until their wavelengths come within the horizon. Therefore, we do not expect back reaction to alter our conclusions, although it may change the instability time scale by factors of order unity. Similarly, we neglect the effect of fluctuations in the matter, since they are coupled to the texture only via gravitation. If these matter fluctuations are correlated or anticorrelated to the fluctuations in the texture field initially, as is often assumed in the texture scenario of structure formation [12], then the amplitude of these fluctuations should be comparable to those of the texture field, and thus their effect would be second order. If these fluctuations are uncorrelated to the texture fluctuation, it might happen that their amplitude is much greater than that of the texture, but in this case it is merely a random fluctuation on the spacetime background, and we would expect that they would not affect the evolution of long wavelength modes of texture.

So, what does this analysis tell us about the requirements for the homogeneity of the initial texture configuration? First of all, we note that all modes are stable, except for the long wavelength $n=1$ curl-free modes and for the divergence-free zero modes which correspond to rotations and translations. From the results in Sec. III, the modes with $\omega^{2}>0$ will decay with the expansion of the universe, so one does not require stringent constraints on the general homogeneity of the initial texture. Since the instability time scale for the unstable modes is comparable to the age of the Universe, the initial inhomogeneity of the texture should be comparable to or less than the inhomogeneity of the universe today. These inhomogeneities can induce perturbations in the metric and further affect the matter distribution, both of which can produce anisotropy in CMB. The isotropy of the CMB should therefore place an upper limit to the acceptable magnitude of this perturbation in the initial scalar-field configuration. Although we have not done a complete analysis of CMB anisotropies induced by this instability, it should give rise to a dipole anisotropy on the sky. The $n=1$ modes have either $l=1$ or $l=0$. The $l=1$ modes are obviously dipole. The $l=0$ mode is isotropic to an observer at the origin, but not to other observers. Indeed, if we make a translation of the origin, the $S^{3}$ spherical harmonics with same $n$, but different $l$ will mix. Thus for a general observer, it appears anisotropic with a dipole pattern. Given that the CMB dipole is $\sim 10^{-3}$, this constrains the magnitude of the initial $\omega^{2}=-1$ perturbation to be less than roughly this value. If the CMB dipole can confidently be aligned with the gradient of the local density field to, say $10 \%$, then the constraint to the initial magnitude of the $\omega^{2}=-1$ perturbation should be an order of magnitude smaller.

We therefore conclude that except for the $\omega^{2}=-1$ modes, inhomogeneities in the scalar-field configuration will decay, so in some sense, the one-texture universe does not require extraordinarily peculiar initial conditions. One only requires that the magnitude of the lowest-eigenmode perturbation be less than $\sim 10^{-3}$. Of course, more precise conclusions regarding the implications for the one-texture universe will have to await a more complete theory of its origin.

\section{ACKNOWLEDGMENTS}

We thank John Barrow, Ruth Durrer, and Erick Weinberg for discussions. M.H. was supported by PPARC and A.R.L. by the Royal Society. This work was supported at Columbia by D.O.E. Contract No. DEFG02-92-ER 40699, NASA NAG5-3091, and the Alfred P. Sloan Foundation. A.R.L. thanks Columbia University for its hospitality during a visit, and M.H., M.K., and A.R.L. thank the University of Uppsala/NORDITA for support during a visit to Sweden. 
[1] N. Turok, Phys. Rev. Lett. 63, 2625 (1989).

[2] R. L. Davis, Phys. Rev. D 35, 3705 (1987).

[3] M. Kamionkowski and N. Toumbas, Phys. Rev. Lett. 77, 587 (1996).

[4] Y. L. Xin, Duke Math. J. 47, 609 (1980); P. F. Leung, in Harmonic Maps, edited by U. R. J. Knill, Lect. Notes Math. Vol. 949 (Springer-Verlag, Berlin, 1982), p. 122; Y. Ohnita, Tôhoku Math. J. 38, 259 (1986).

[5] M. Kamionkowski and K. Freese, Phys. Rev. Lett. 69, 2743 (1992).

[6] C. W. Misner, Phys. Rev. D 18, 4510 (1978).
[7] I. S. Gradshteyn and I. M. Ryzhik, Table of Integrals, Series, and Products (Academic, New York, 1994).

[8] L. P. Grishchuk, Zh. Éksp. Teor. Fiz. 67, 823 (1974) [Sov. Phys. JETP 40, 409 (1974)].

[9] M. Abramowitz and I. A. Stegun, Handbook of Mathematical Functions (Dover, New York, 1972).

[10] B. Allen, Phys. Rev. D 37, 2078 (1988).

[11] V. D. Sandberg, J. Math. Phys. (N.Y.) 19, 2441 (1978).

[12] See, for example, U. Pen, D. N. Spergel, and N. Turok, Phys. Rev. D 49, 692 (1994). 